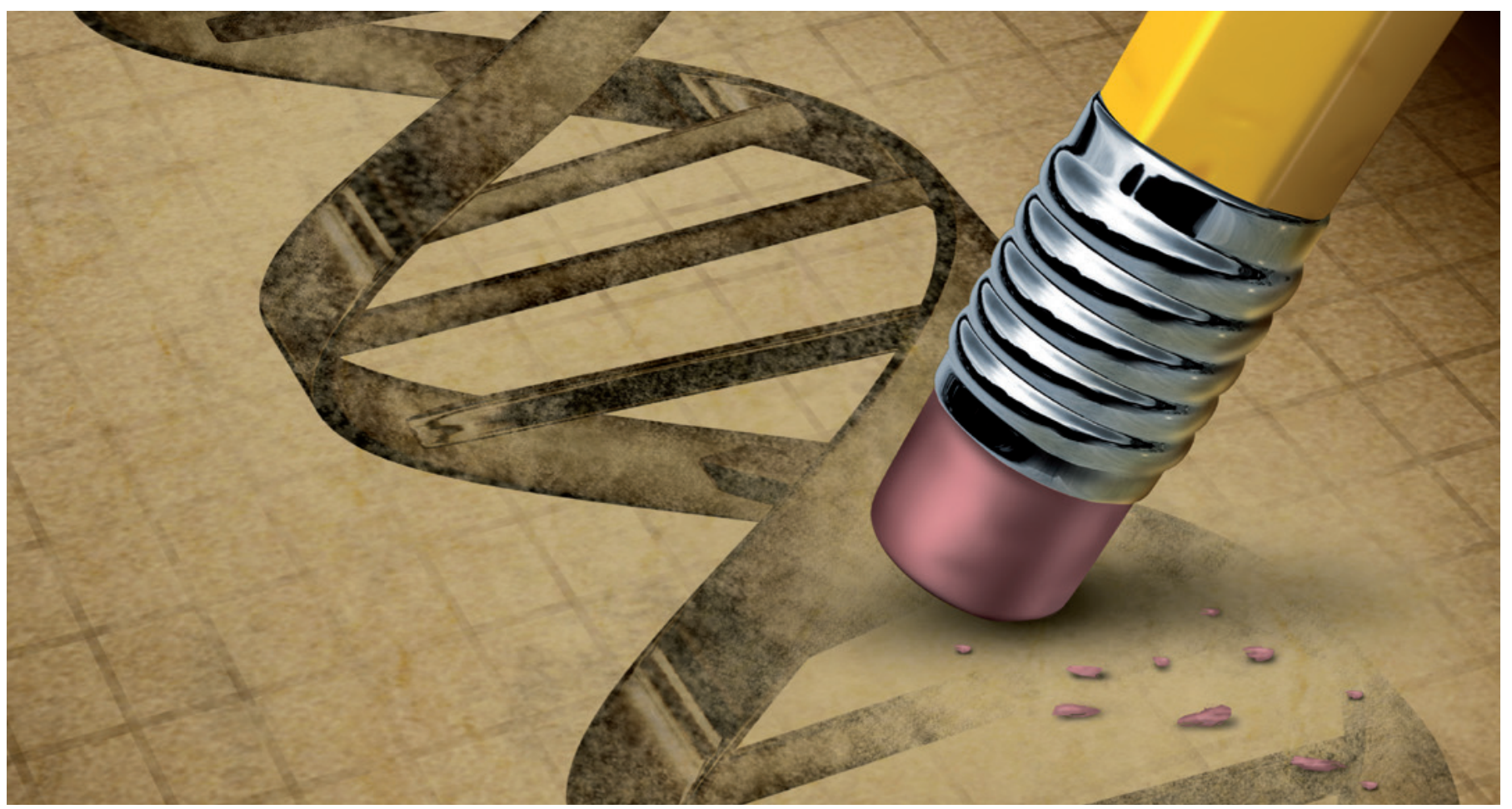

\title{
Don't edit the human germ line
}

\section{Heritable human genetic modifications pose serious risks, and the therapeutic benefits are tenuous, warn Edward Lanphier, Fyodor Urnov and colleagues.}

$\mathrm{I}$ $\mathrm{t}$ is thought that studies involving the use of genome-editing tools to modify 1 the DNA of human embryos will be published shortly ${ }^{1}$.

There are grave concerns regarding the ethical and safety implications of this research. There is also fear of the negative impact it could have on important work involving the use of genome-editing techniques in somatic (non-reproductive) cells.

We are all involved in this latter area of work. One of us (F.U.) helped to develop the first genome-editing technology, zinc-finger nucleases $^{2}$ (ZFNs), and is now senior scientist at the company developing them, Sangamo BioSciences of Richmond, California. The Alliance for Regenerative Medicine (ARM; in which E.L., M.W. and S.E.H. are involved), is an international organization that represents more than 200 life-sciences companies, research institutions, non-profit organizations, patient-advocacy groups and investors focused on developing and commercializing therapeutics, including those involving genome editing.
Genome-editing technologies may offer a powerful approach to treat many human diseases, including HIV/AIDS, haemophilia, sickle-cell anaemia and several forms of cancer ${ }^{3}$. All techniques currently in various stages of clinical development focus on modifying the genetic material of somatic cells, such as $T$ cells (a type of white blood cell). These are not designed to affect sperm or eggs.

In our view, genome editing in human embryos using current technologies could have unpredictable effects on future generations. This makes it dangerous and ethically unacceptable. Such research could be exploited for non-therapeutic modifications. We are concerned that a public outcry about such an ethical breach could hinder a promising area of therapeutic development, namely making genetic changes that cannot be inherited.

At this early stage, scientists should agree not to modify the DNA of human reproductive cells. Should a truly compelling case ever arise for the therapeutic benefit of germline modification, we encourage an open discussion around the appropriate course of action.

\section{EDITING TOOLS}

Genome editing of human somatic cells aims to repair or eliminate a mutation that could cause disease. The premise is that corrective changes to a sufficient number of cells carrying the mutation - in which the genetic fixes would last the lifetimes of the modified cells and their progeny - could provide a 'one and done' curative treatment for patients.

For instance, ZFNs are DNA-binding proteins that can be engineered to induce a double-strand break in a section of DNA. Such molecular scissors enable researchers to 'knock out' specific genes, repair a mutation or incorporate a new stretch of DNA into a selected location.

Sangamo BioSciences is conducting clinical trials to evaluate an application of genome editing as a potential 'functional cure' for HIV/AIDS ${ }^{4}$. The hope is that 
intravenous infusion of modified T cells will enable patients to stop taking antiretroviral drugs. A phase I trial in patients with $\beta$-thalassaemia - a genetic blood disorder caused by insufficient haemoglobin production - is scheduled to begin this year.

The newest addition to the genomeediting arsenal is CRISPR/Cas9, a bacteriaderived system that uses RNA molecules that recognize specific human DNA sequences. The RNAs act as guides, matching the nuclease to corresponding locations in the human genome. CRISPR/Cas9 is the simplest genome-editing tool to work with because it relies on RNA-DNA base pairing, rather than the engineering of proteins that bind particular DNA sequences.

The CRISPR technique has dramatically expanded research on genome editing. But we cannot imagine a situation in which its use in human embryos would offer a therapeutic benefit over existing and developing methods. It would be difficult to control exactly how many cells are modified. Increasing the dose of nuclease used would increase the likelihood that the mutated gene will be corrected, but also raise the risk of cuts being made elsewhere in the genome.

In an embryo, a nuclease may not necessarily cut both copies of the target gene, or the cell may start dividing before the corrections are complete, resulting in a genetic mosaic. Studies using gene-editing in animals such as rats ${ }^{5}$, cattle ${ }^{6}$, sheep ${ }^{7}$ and pigs $^{8}$, indicate that it is possible to delete or disable genes in an embryo - a simpler process than actually correcting DNA sequences — in only some of the cells.

The current ability to perform quality controls on only a subset of cells means that the precise effects of genetic modification to an embryo may be impossible to know until after birth. Even then, potential problems may not surface for years. Established methods, such as standard prenatal genetic diagnostics or in vitro fertilization (IVF) with the genetic profiling of embryos before implantation, are much better options for parents who both carry the same mutation for a disease.

\section{LEGAL CASE}

Patient safety is paramount among the arguments against modifying the human germ line (egg and sperm cells). If a mosaic embryo is created, the embryo's germ line may or may not carry the genetic alteration. But the use of CRISPR/Cas9 in human embryos certainly makes onward human germline modification a possibility. Philosophically or ethically justifiable applications for this technology - should any ever exist - are moot until it becomes possible to demonstrate safe outcomes and obtain

reproducible data over multiple generations.

Because of such concerns - as well as for serious ethical reasons - some countries discouraged or prohibited this type of research a decade before the technical feasibility of germline modification was confirmed in rats in 2009 (ref. 9). (Today, around 40 countries discourage or ban it.)

Many countries do not have explicit legislation in place permitting or forbidding genetic engineering in humans - considering such research experimental and not therapeutic (see go.nature.com/uvthmu). However, in nations with policies regarding inheritable genetic modification, it has been prohibited by law or by measures having the force of law.

This consensus is most visible in western Europe, where 15 of 22 nations prohibit the modification of the germ line ${ }^{4}$. Although the United States has not offi-

"The precise
effects of
genetic
modification
to an embryo
may be
impossible
to know until
after birth."
cially prohibited germline modification, the US National Institutes of Health's Recombinant DNA Advisory Committee explicitly states that it "will not at present entertain proposals for germ line alterations" (see go.nature.com/mgscb2).

In general, researchers who want to investigate the clinical uses of genetically engineered somatic cells must secure people's informed consent. In the United States, this takes place under the oversight of the Food and Drug Administration and the Department of Health and Human Services. For research involving genetic modification of the germ line, it is unclear what information would be needed - or obtainable - to adequately inform prospective parents of the risks, including to future generations.

Many oppose germline modification on the grounds that permitting even unambiguously therapeutic interventions could start us down a path towards non-therapeutic genetic enhancement. We share these concerns.

\section{DIALOGUE NEEDED}

Ten years ago, the Genetics and Public Policy Center, now in Washington DC, brought together more than 80 experts from the United States and Canada to consider the scientific and ethical consequences of genetically modifying the human germ line. Now that the capability for human germline engineering has emerged, we urge the international scientific community to engage in this type of dialogue. This is needed both to establish how to proceed in the immediate term, and to assess whether, and under what circumstances - if any future research involving genetic modification of human germ cells should take place.
Such discussions must include the public as well as experts and academics.

An excellent precedent for open, early discussion as new scientific capabilities emerge was set by the hearings, consultations and reports involving scientists, bioethicists, regulators and the general public that preceded the UK government's decision to legalize mitochondrial DNA transfer in February. We are not, of course, making a comparison between the replacement of faulty mitochondrial DNA in an egg or embryo with healthy DNA from a female donor and the use of genome-editing in human embryos. In mitochondrial transfer, the aim is to prevent life-threatening diseases by replacing a known and tiny fraction of the overall genome.

Key to all discussion and future research is making a clear distinction between genome editing in somatic cells and in germ cells. A voluntary moratorium in the scientific community could be an effective way to discourage human germline modification and raise public awareness of the difference between these two techniques. Legitimate concerns regarding the safety and ethical impacts of germline editing must not impede the significant progress being made in the clinical development of approaches to potentially cure serious debilitating diseases.

Edward Lanphier is president and chief executive officer of Sangamo BioSciences in Richmond, California, USA, and chairman of the Alliance for Regenerative Medicine in Washington DC, USA. Fyodor Urnov is senior scientist at Sangamo BioSciences in Richmond, California, USA. Sarah Ehlen Haecker is director of technology sections at the Alliance for Regenerative Medicine in Washington DC, USA. Michael Werner is executive director of the Alliance for Regenerative Medicine in Washington DC, USA. Joanna Smolenski is a PhD student in philosophy at the Graduate Center of the City University of New York, New York, USA.

e-mail:lanphier@sangamo.com

1. Regalado, A. MIT Tech. Rev. http://go.nature. com/2n2nfl (2015).

2. Urnov, F. D., Rebar, E. J., Holmes, M. C.,

Zhang. H. S. \& Gregory, P. D. Nature Rev. Genet. 11, 636-646 (2010).

3. Carroll, D. Annu. Rev. Biochem. 83, 409-439 (2014).

4. Tebas, P. et al. N. Engl. J. Med. 370, 901-910 (2014).

5. Yoshimi, K., Kaneko, T., Voigt, B. \& Mashimo, T. Nature Commun. 5, 4240 (2014).

6. Heo, T. Y. et al. Stem Cells Dev. 24, 393-402 (2015).

7. Han, H. et al. Front. Agr. Sci. Eng. 1, 2-5 (2014)

8. Kang, Q. et al. in Proc. 10th World Congr. Genet. Appl. Livestock Prod. http://go.nature. com/6mtz2d (2014).

9. Geurts, A. M. Science. 325, 433 (2009).

The authors declare competing financial interests, see go.nature.com/plkopd for details. 